\title{
Pattern of Constipation and Response to Polyethylene Glycol in Children
}

\author{
Saha $\mathbf{S}^{1}$, Bose $\mathrm{K}^{2}$, Das $\mathrm{K}^{3}$, Mridha $\mathrm{D}^{4}$, Das $\mathrm{I}^{5}$, Mondal $\mathrm{P}^{6}$
}

${ }^{1}$ Dr. Sudip Saha MBBS. MD, Associate Professor, ${ }^{2} \mathrm{Dr}$. Kallol Bose, MBBS. MD Assistant Professor, ${ }^{3}$ Dr. Kallol Das, MBBS. MD RMO cum Tutor, ${ }^{4} \mathrm{Dr}$. Dhrubojyoti Mridha, MBBS. MD Assistant Professor, ${ }^{5} \mathrm{Dr}$. Ira Das, MBBS. MD RMO cum Tutor, ${ }^{6}$ Dr. Piyasi Mondal, MBBS. DNB RMO cum Tutor. All from the Department of Paediatrics, Chittaranjan Seva Sadan, Sonapur, Kolkata, India.

\section{Address for correspondence:}

Dr. Kallol Bose

Desbandhu Park Sonarpur

Kolkata700150,

West Bengal, INDIA

Rel No;+9109836653608

E-mail: subhosrisaha@gmail.com

Acknowledgements: None

Funding: Nil

Conflict of Interest: None

Permission from IRB: Yes

Ethical dilemmas faced during study: No

\section{How to cite}

Saha S, Bose K, Das K, Mridha D, Das I, Mondal P. Pattern of Constipation and Response to Polyethylene Glycol in Children. J Nepal Paediatr Soc 2016;36(3):263-267.

doi: http://dx.doi.org/10.3126/jnps.v36i3.15726

This work is licensed under a Creative Commons Attribution 3.0 License.

\begin{abstract}
Introduction:Constipation is a common problem in children with worldwide prevalence between $0.7 \%$ and $29.6 \%$. Materials and Methods:Total number of children was 232 out of which 16 were discarded due to loss in follow up. Inclusion criteria: Any child aged one month to ten years presenting with constipation. We used NASPGHAN definition of constipation. Exclusion criteria: Critically sick and hemodynamically unstable patients were excluded from the study. Data were collected for age, sex, duration of constipation, symptoms and signs such as stool frequency, stool consistency, pain during defecation, presence of blood in stool, fecal and urinary incontinence, and presence of fecal impaction or an abdominal mass. Clinical evaluation (history and physical examination) of all patients was done by the same physician to avoid bias in clinical finding. Polyethylene glycol (PEG) was tried in all patients in a dose of 0.3 to $2.1 \mathrm{~g} / \mathrm{kg}$. Response was defined as passage of at least one semisolid stool without discomfort with use of PEG for at least 4 weeks. Results: In our prospective study done on 232 patients with constipation over three year period (dividing patient's into 1 month to 5 months, 6 months to 5 year and 6 years to 10 years) revealed that constipation is mostly prevalent in 6 months to 5 year age group with slight male preponderance. Most of them had symptom onset after six months of age. Commonest symptom was hard stool in general $(79.6 \%)$ but prevalence of pain abdomen increases with age and peaks in above five year group. Commonest sign is palpable fecal mass. Complications-urinary dysfunction, fecal incontinence, fissure are common in older age group. Functional constipation was the commonest cause (96.2\%). Hirschprung disease was diagnosed in $1.4 \%$. Polyethylene glycol shows good response in above 6 months of age groups. Conclusion: Functional constipationis the commonest cause of constipation. Mostly affected group is six months to five years. Polyethelene Glycol is an effective treatment especially after six months.
\end{abstract}

Key words: Polyethylene glycol 


\section{Introduction}

C onstipation is a common problem in children. The worldwide prevalence varies between $0.7 \%$ and $29.6 \%{ }^{1}$. Constipation is the reason for $3 \%$ to $5 \%$ of physician visits by children ${ }^{2}$. Constipation has a significant impact on the use and cost of medical services $^{3}$ Childhood constipation affects children's physical, social, emotional and school functioning ${ }^{4}$. As normal bowel habits vary with age ${ }^{5}$ prevalence and symptoms of constipation are often different in different age groups. Constipation peaks at the age of toilet training ${ }^{6}$.

A longer duration of constipation before diagnosis has been associated with complications (e.g., fecal incontinence) and poorer long-term outcome (persistence of symptoms and continuous need for laxatives $)^{6}$.Constipation is defined as a feeling of unsatisfactory stools -could be too hard stool, passage of too small stool or too difficult to expel ${ }^{7}$. The guidelines of the North American Society of Pediatric Gastroenterolgy, Hepatology and Nutrition (NASPGHAN) defines constipation as a delay or difficulty in defecation present for two or more weeks which causes significant distress to the patient.

Causes of constipation can be divided into primary (functional) and secondary causes. Functional constipation means constipation without an underlying organic abnormality, drug-induced, endocrine abnormality or anatomical defect. Functional or nonorganic causes are responsible for $85-95 \%$ of chronic constipation in children while organic causes are seen in $5-15 \%$ of cases. This prospective study intends to reveal the prevalence of constipation in three different age groups ( 1 month to 5 months, 6 months to 5 years and 6 to 10 years) and also gender distribution among the aforesaid age groups. This study also describes the different clinical features, investigation findings, diagnosis including organic and functional causes and response to polyethylene glycol (PEG) in above mentioned age groups.

The aim of this study was to find out the pattern of constipation in children attending a tertiary care hospital.

\section{Material and Methods}

Children with constipation who visited our outpatient department at ChittaranjanSevaSadan Kolkata from April 2012 to May 2015 were included in the study. Total number of children were 232 out of which 16 were excluded due to loss in follow up.
Patients were recruited according to following inclusion and exclusion criteria. Inclusion criteria: Children one month to ten years age presenting with constipation. Constipation was defined as per the NASPGHAN definition which states that"constipation is a delay or difficulty in defecation, present for two weeks or more, and sufficient to cause significant distress to the patient", was followed.However fewer than three bowel motions per week is a commonly used definition of constipation and were reported by only few patients. Since, using only this definition would lead to under-diagnosis of constipation $^{8}$ so hard, dry stool and painful defecation appeared to be more sensitive indicators ${ }^{8}$.Exclusion criteria: Critically sick and hemodynamically unstable patients were excluded from the study.

Relevant history was taken from either the patient if appropriate or from the patient's caregiver in prepared sheet containing specific information. Data collected included age, sex, duration of constipation, symptoms and signs such as bowel frequency and consistency, painful defecation, presence of bloody stool, fecal and urinary incontinence, presence of fecal impaction or an abdominal mass. Clinical evaluation (history and physical examination) of all patients was done by the same physician to avoid bias in clinical finding. Digital rectal examination was deferred in cases of active anal fissures.

Laboratory and radiological investigations were performed according to the patient's presentation. Barium enema, thyroid profile, Celiac serology were done on all cases on wheat based diet. MRI spine, rectal manometry, and full thickness rectal biopsy were done in selected cases. Hirschsprung's disease was confirmed only after rectal biopsy. Cow's milk protein intolerance was considered in children, if elimination of cow's milk formula resulted in improvement; and if recurrence of symptoms was seen after reintroduction of cow's milk by oral challenge. Neurological disorders were diagnosed with clinical and appropriate investigations.

For data analysis and comparison, we categorized the patients according to gender and age into three groups. Group I-(1 to 5 months), Group II- (6 months to 5 years), Group III- (6 years to 10 years).

Faecal incontinence also known as encopresis or soiling, refers to the repetitive, involuntary, inappropriate passage of stool by a child after attaining complete bowel control $^{9}$ Children who attained faecal control before 24 months were not considered. Polyethylene glycol (PEG) was tried in all patients in a dose of 0.3 
to $2.1 \mathrm{~g} / \mathrm{kg}^{10}$. We started with a dose of $0.3 \mathrm{mg} / \mathrm{kg}$ and escalated every sixth day until maximum dose of PEG was achieved. Response was defined as passage of at least one semisolid stool without discomfort with use of PEG for at least four weeks. Data were tabulated and statistical analyses were performed with help of Epi Info (TM) 3.5.3. EPI INFO is a trademark of the Centers for Disease Control and Prevention (CDC). Using this software, basic cross-tabulation, inferences and associations were performed, $p$-values $<0.05$ were considered significant.

Approval from Institutional Ethical Committee was taken prior to the study. Informed consents were taken in standard performa after proper counselling of legal guardians and patients, as required.

\section{Results}

During our study period, in our clinic 232 had constipation, 216 patients were included in the analysis after exclusion of 16 patients. Patient's distribution was done by age, sex, onset of symptoms, clinical symptoms and signs, investigationsand treatment response to polyethylene glycol (PEG). Of the 216 patients, $13 \%$ was in the age group I, $66.7 \%$ in age group II, and $20.4 \%$ in age group III. Mostly affected was group II. In general amongst all patients $59.3 \%$ $(n=128)$ of patients were male and $40.7 \%(n=88)$ were female. $83.3 \%$ of patients had onset of symptoms after six months age, only $16.7 \%$ before six months of age. In general commonest symptom was passage of hard stool $(79.6 \%)$ followed by pain abdomen (59.3\%) excluding children below one year who cannot express pain abdomen properly. Faecal incontinence (18.5\%), passage of pellet like stool $(9.3 \%)$ and urinary voiding dysfunction (7.4\%). Pain abdomen was mostly prevalent in the age group III (81.8\% of patients) followed by age group II (63.9\%).
Hard stool was complained in $94.4 \%$ of patients of age group II, $57.1 \%$ in age group I and $45.5 \%$ of age group III. As the patients in age group I cannot express pain so hard stool is the major feature. In the group age II hard stool is the major symptom compared to pain abdomen (94.4\% vs $63.9 \%$ ) but the picture is just the reverse in age group III (45.5\% vs $81.8 \%$ ). Commonest sign was palpable fecal mass seen in $16.7 \%$ of patients. Anal fissure was detected in $14.8 \%$ of patients. During per rectal examination $92.6 \%$ patients had loaded rectum and $7.4 \%$ cases rectum was empty. $1.3 \%$ patients showed facial dysmorphism. About 96.2\% patients was diagnosed to have functional constipation $(n=208)$, Hirschprung's disease (HD)was diagnosed in $1.4 \%$ of patients $(n=3)$ hypothyroidism was diagnosed in $1.38 \%(n=3)$ of patients, local neurologic problem in $0.9 \%(n=2)$. Before coming to our set up only $14.8 \%$ of patients were treated with medicine for more than eight weeks, rest were treated irregularly or prolonged treatment was not given.

Response to PEG was quite good in both age group II and III (91.7\%, 90.9\% respectively). In age group I response was only $42.9 \%$. $90 \%$ of cases of functional constipation responded well, only $33.3 \%(n=1$ out of total 3 , but recurrence with stoppage or reduction of dose) of HD and the only hypothyroid patient did not respond to PEG. Barium enema showing delayed transit of contrast in $14.8 \%(n=32)$ of cases. Patients whose per rectal examination revealed empty rectum $(n=16)$, patient not responding to $P E G(n=32)$, and whose barium study shows delayed transit of dye $(n=32)$ were selected for rectal manometry (total 44). 4 of them showed very high pressure for opening of anal sphincter-out of which three was histologically diagnosed to have HD (Table 1 and 2).

Table 1: Clinical features of constipation

\begin{tabular}{lccc}
\hline & Age group I & Age group II & Age group III \\
\hline Pain abdomen \% & ---- & 63.9 & 81.8 \\
\hline Hard stool \% & 57.1 & 94.4 & 45.5 \\
\hline Pellet Stool \% & 28.6 & 8.3 & 0.0 \\
\hline Fecal Incontinence \% & ---- & 5.6 & 63.6 \\
\hline UVD \% & ----- & 5.6 & 18.2 \\
\hline Fecal Mass \% & 28.6 & 11.1 & 27.3 \\
\hline Fissure \% & 0.0 & 8.3 & 45.5 \\
\hline
\end{tabular}


Table 2: Etiologic Diagnosis in different age groups

\begin{tabular}{lccc}
\hline Diagnosis & $\begin{array}{c}\text { Age group I } \\
(\mathbf{n}=\mathbf{2 8})\end{array}$ & $\begin{array}{c}\text { Age group II } \\
(\mathbf{n}=\mathbf{1 4 4})\end{array}$ & $\begin{array}{c}\text { Age group III } \\
(\mathbf{n}=\mathbf{4 4})\end{array}$ \\
\hline Hirschprung's Disease & 1 & 1 & 1 \\
\hline Hypothyroid & 2 & 0 & 1 \\
\hline Dysmorphic & 1 & 2 & 0 \\
\hline Functional constipation & 22 & 141 & 42 \\
\hline Neuro-deficit & 2 & 0 & 0 \\
\hline
\end{tabular}

\section{Discussion}

We conducted this prospective study to find out the pattern of constipation in children attending our tertiary care hospital. Our study showed that constipation is mostly prevalent in group II similar to previous studies ${ }^{8}$. Preschool children were the most commonly affected age group. Most of children with constipation the age group II described their bowel motions as hard. This is consistent with the work of Loening-Baucke ${ }^{11}$, who opined that using stool consistency and pain during defecation to define constipation is more sensitive than using duration of symptoms or frequency of stool. Passage of hard stool was the predominant feature overall but in children above five years; pain abdomen was the major symptom. We did not take the history of incontinence of stool before three years.

In our study overall $18.5 \%$ of patients had fecal incontinence but in age group III incidence was as high as $63.6 \%$. Retentive fecal incontinence (associated with constipation) has been reported in up to $85 \%$ of constipated children in previous studies ${ }^{9}$. The urinary system in human is adjacent to the gut and it is also under same neurological control. Rectal pathology causes urinary symptoms through mechanical pressure of the stool over the urinary bladder. Pelvic floor muscle spasm causes urinary voiding dysfunction. Constipation may cause urinary tract infections, vesico-ureteric reflux (VUR) and enuresis as a result of uninhibited bladder contraction ${ }^{12,13}$. Urinary symptoms were seen in $7.4 \%$ of patients in our study i.e. consistent with previous studies ${ }^{14}$. Urinary voiding problem, fecal incontinence and fissure were highest in above five year age group $(18.2 \%, 63.6 \%$ and $45.5 \%$ respectively). In previous studies pain abdomen was reported in about onethird of children with constipatation ${ }^{15}$. In our cohort, almost $59.3 \%$ of our patients had pain abdomen, and the prevalence rate is high in higher age. The lower incidence in younger age may reflect under-reporting.

On physical exam, a fecal mass in the rectum was the commonest finding in all ages. Fecal mass was detected in $28.6 \%$ of patients of age group I, $11.1 \%$ of age group II and $27.3 \%$ of age group III. Regarding the underlying cause of constipation, functional constipation was the commonest cause of chronic constipation (96.2\%). In each age group HD (1.4\%) was diagnosed (one for each group) - this is definitely lower than other studies $^{16}$. Response to PEG was not satisfactory in less than 6 months age group. But beyond that it works very well.

\section{Conclusion}

This is the study describing the clinical profile of pediatric constipation in children according to age group. The clinical characteristics are different in three age groups. Predominant affected group was six months to five years. Commonest cause of constipation was functional. Hard stool was the commonest presentation in under five children. Pain abdomen was predominant feature in above five year children. PEG is very much effective in functional constipationespecially after six months

Limitations:The results of this study are limited by the prospective nature of the study and small number of adolescents in this cohort also limits the generalization of our results to this paediatric population. 


\section{References}

1. Van den Berg MM, Benninga MA, Di Lorenzo C. Epidemiology of childhood constipation: a systematic review. Am J Gastroenterol2006;101:2401-409.

2. Loening-Baucke V. Chronic constipation in children. Gastroenterology1993;105:1557-564.

3. Liem O, Harman J, Benninga M, Kelleher K, Mousa $\mathrm{H}$, Di Lorenzo C. Health utilization and cost impact of childhood constipation in the United States. J Pediatr2009;154:258-62.

4. Rajindrajith S, Devanarayana NM, Weerasooriya L, Hathagoda W, Benninga MA. Quality of life and somatic symptoms in children with constipation: a school-based study. J Pediatr2013;163:1069-72.

5. Weaver LT, Steiner $H$. The bowel habit of young children. Arch Dis Child1984;59:649-52.

6. Loening-Baucke V. Constipation in early childhood: patient characteristics, treatment, and longterm follow up. Gut1993;34:1400-404

7. Baker SS, Liptak GS, Colletti RB, Croffie JM, Di Lorenzo C, Ector W, et al. Constipation in infants and children: evaluation and treatment. A medical position statement of the North American Society for Pediatric Gastroenterology and Nutrition. J PediatrGastroenterolNutr 1999;29:612-26.

8. Altamimi E. Clinical Charecteristics of Pediatric Constipation in South Jordan. PediatrGastroenterolHepatolNutr 2014;17(3):155-61. doi: 10.5223/pghn.2014.17.3.155
9. Rajindrajith S, Devanarayana NM, Benninga MA Review article: faecal incontinence in children: epidemiology, pathophysiology, clinical evaluation and management. AlimentPharmacolTher 2013;37:37-48.

10. Loening-Baucke V, Krishna R, Pashankar DS Polyethylene glycol 3350 without electrolytes for the treatment of functional constipation in infants and toddlers. JPediatrGastroenterolNutr2004;39(5):536-39.

11. Loening-Baucke V. Prevalence, symptoms and outcome of constipation in infants and toddlers. $J$ Pediatr2005;146:359-63.

12. Di Lorenzo C. Approach to the child with constipation and encopresis. In: Rudolph CD, Rudolph MR, editors. Rudolph's pediatrics United States of America: McGraw-Hill; 2002. pp. 1368-1370.

13. Loening-Baucke V. Urinary incontinence and urinary tract infection and their resolution with treatment of chronic constipation of childhood. Pediatrics1997;100:228-32.

14. Baker SS, Liptak GS, Colletti RB, Croffie JM, Di Lorenzo C, Ector W, et al. Constipation in infants and children: evaluation and treatment. A medical position statement of the North American Society for Pediatric Gastroenterology and Nutrition. J PediatrGastroenterolNutr1999;29:612-26.

15. Afzal NA, Tighe MP, Thomson MA. Constipation in children. Ital J Pediatr2011;37:28.

16. Khanna V, Poddar U, Yachha SK. Etiology and Clinical Spectrum of Constipationin Indian Children. Indian Pediatr 2010;47:1025-30. 